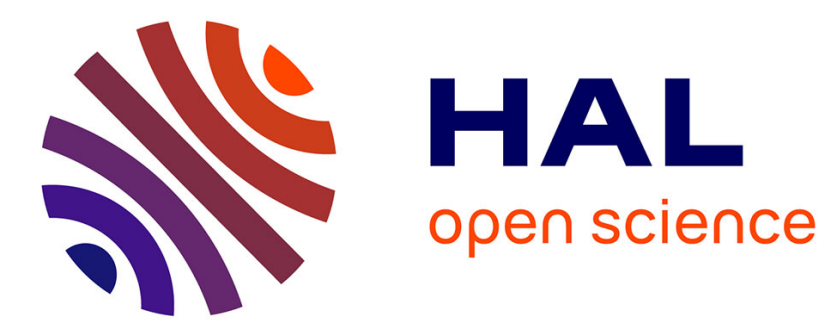

\title{
Variomètre sensible à aimant pour la mesure de la déclinaison
}

\author{
J. Mosnier
}

\section{To cite this version:}

J. Mosnier. Variomètre sensible à aimant pour la mesure de la déclinaison. Revue de Physique Appliquée, 1970, 5 (1), pp.151-152. 10.1051/rphysap:0197000501015100 . jpa-00243350

\section{HAL Id: jpa-00243350 https://hal.science/jpa-00243350}

Submitted on 1 Jan 1970

HAL is a multi-disciplinary open access archive for the deposit and dissemination of scientific research documents, whether they are published or not. The documents may come from teaching and research institutions in France or abroad, or from public or private research centers.
L'archive ouverte pluridisciplinaire HAL, est destinée au dépôt et à la diffusion de documents scientifiques de niveau recherche, publiés ou non, émanant des établissements d'enseignement et de recherche français ou étrangers, des laboratoires publics ou privés. 


\title{
VARIOMÈTRE SENSIBLE A AIMANT POUR LA MESURE DE LA DÉGLINAISON
}

\author{
Par J. MOSNIER, \\ École Normale Supérieure, Paris (France).
}

\begin{abstract}
Résumé. - Les performances des variomètres classiques destinés à la mesure de $D$ peuvent être améliorées par l'emploi d'une contre-réaction de champ qui transforme le magnétomètre en appareil de zéro. A long terme, leur sensibilité est cependant limitée vers $0,2 \gamma$ par des dérives thermiques, principalement dans le détecteur d'écart photoélectrique et l'amplificateur électronique qui lui fait suite. L'appareil décrit utilise une technique de modulation de lumière qui permet d'éviter ces deux inconvénients. La sensibilité est alors bien meilleure, de l'ordre de $0,01 \gamma$ pour des phénomènes de période comprise entre quelques secondes et quelques heures.
\end{abstract}

Abstract. - The performances of the classical variometers destined to measure $D$ can be improved by the utilisation of a negative feedback which transforms the magnetometer into a null detector. Over a long period, their sensibility is however limited towards $0.2 \gamma$ by thermal drifts, principally in the photoelectric differential detector and the electronic amplifier which follows it. The apparatus described uses a technique of light modulation which allows these two inconveniences to be avoided. The sensibility is then much better, in the order of $0.01 \gamma$ for the period phenomena between several seconds and a few hours.

L'appareil décrit ci-dessous est destiné à l'étude des très petites variations de la déclinaison $D$ du champ magnétique terrestre.

La figure 1 représente un variomètre de déclinaison

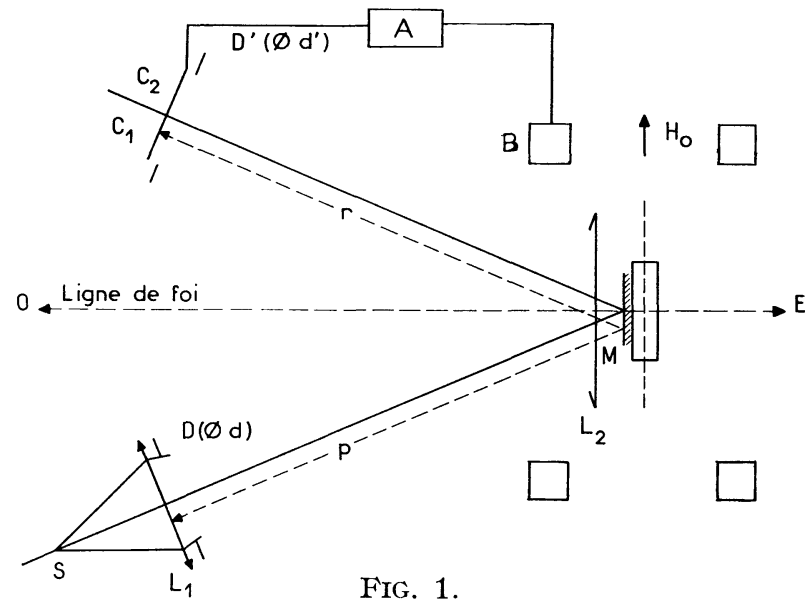

classique à aimant asservi. Il est composé d'un aimant suspendu à un fil sans torsion, d'un système optique $\mathrm{S}$, $\mathrm{L}_{1}, \mathrm{~L}_{2}$, et d'un détecteur photoélectrique formé de deux cellules $\mathrm{C}_{1} \mathrm{C}_{2}$ montées en opposition. Si l'aimant tourne d'un angle $\varepsilon$ à partir d'une direction de référence située dans le plan de symétrie du détecteur, il apparaît aux bornes de $\mathrm{C}_{1} \mathrm{C}_{2}$ une tension proportionnelle à l'écart qui, amplifiée en $\mathrm{A}$, produit dans des bobines de Helmholtz B entourant l'aimant un champ de contre-réaction. En boucle fermée, l'aimant prend alors une direction pratiquement fixe dans l'espace.

Dans ces conditions, on peut écrire, en appelant $\Delta D$ une variation naturelle de la déclinaison et $\Delta D^{\prime}$ la variation correspondante créée au centre des bobines B par le système d'asservissement :

$$
\Delta D^{\prime}=\Delta D\left(\frac{A}{1+A}\right)
$$

$A$ étant le gain de la chaîne en boucle ouverte.
On voit que, si $A$ est suffisamment grand, $\Delta D^{\prime}$ est sensiblement équivalent à $\Delta D$, et l'enregistrement du champ $\Delta D^{\prime}$ (proportionnel au courant traversant les bobines B) permettra l'étude des variations naturelles de la déclinaison.

En toute rigueur, on doit écrire la relation (1) sous la forme :

$$
\Delta D^{\prime}=\Delta D\left(\frac{A}{1+A}\right)+B .
$$

Le terme $B$ correspond à des phénomènes aléatoires d'origine non magnétique que nous appellerons « bruit » s'il s'agit de phénomènes à court terme et « dérive » s'il s'agit de variations lentes.

Une analyse du fonctionnement du magnétomètre montre que la principale source de fluctuations rapides est le bruit de scintillation du détecteur photoélectrique. Ce type de bruit possède un spectre d'énergie, par cycle de bande passante, qui décroît avec la fréquence jusqu'à environ $1000 \mathrm{c} / \mathrm{s}$. Dans la bande $0-1 \mathrm{c} / \mathrm{s}$, il limite la sensibilité du magnétomètre à $0,02 \gamma$ environ.

La principale cause de dérive est la variation, avec la température $\theta$, de la sensibilité $\sigma$ des cellules photoélectriques, et, dans une moindre mesure, la dérive, également d'origine thermique, de l'amplificateur continu d'asservissement. Ces deux causes cumulées peuvent entraîner des variations au niveau de l'entrée du magnétomètre de l'ordre de $0,02 \gamma /{ }^{\circ} \mathrm{C}$ et, si l'on admet que $\theta$ peut varier d'une dizaine de degrés au cours de la journée, limite la sensibilité de l'appareil à $0,2 \gamma$ environ sur 24 heures.

Pour améliorer ces performances, nous avons d'abord utilisé une lumière modulée. A cet effet, la source lumineuse est alimentée par une tension en créneau dont la valeur est alternativement 0 et $V$, la fréquence de modulation étant voisine de $80 \mathrm{c} / \mathrm{s}$. Dans ces conditions, la lumière renferme une composante variable, de forme triangulaire, en retard de $\pi / 2$ sur la tension de modulation, la variation instantanée de l'intensité représentant environ $20 \%$ de celle de la source. 
Les circuits électroniques comprennent ( fig. 2) un oscillateur associé à un amplificateur de puissance alimentant la source et un comparateur de phase qui reçoit d'une part un signal de référence retardé de $\pi / 2$

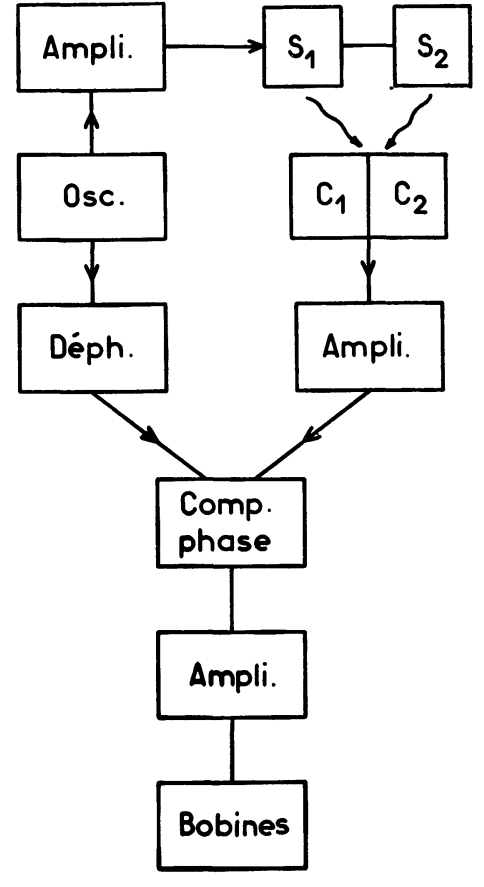

FIG. 2.

par rapport à celui de l'oscillateur, et d'autre part le signal photoélectrique amplifié environ 300000 fois grâce à l'amplificateur $\mathrm{A}$. A la sortie du comparateur de phase, on obtient un signal continu qui s'annule quand la direction du faisceau lumineux de retour coïncide avec celle de référence et dont le signe dépend de la position relative de ces deux directions. Cette dernière tension amplifiée par un étage continu $\mathrm{A}^{\prime}$ sert à alimenter les bobines d'asservissement.

Un tel dispositif améliore les performances du magnétomètre précédemment décrit en transposant autour de la fréquence $80 \mathrm{c} / \mathrm{s}$ l'information initialement contenue autour de la fréquence 0 . En raison de la distribution spectrale de l'énergie dans le bruit de scintillation, on augmente ainsi le rapport signal/bruit du détecteur malgré la perte due à la modulation incomplète de la lumière. On élimine aussi les dérives de l'amplificateur A. En revanche, le dispositif est sans influence sur celles produites par la variation de la sensibilité des cellules avec leur température.

Pour faire disparaître cette deuxième cause de dérives, nous utilisons le dispositif de la figure 3 . Il comporte deux sources lumineuses distinctes $\mathrm{S}_{1}$ et $\mathrm{S}_{2}$ toutes deux modulées de la même manière mais en opposition de phase. La lumière issue de $S_{1}$ se réfléchit comme précédemment sur un miroir $M_{1}$ porté par l'aimant, tandis que celle issue de $\mathrm{S}_{2}$ se réfléchit sur un miroir fixe $\mathbf{M}_{2}$ orienté exactement Nord-Sud. Les réglages sont faits de manière que, lorsque l'aimant est parallèle au miroir fixe, les cellules $\mathrm{C}_{1}$ et $\mathrm{C}_{2}$ sont également éclairées à la fois par $\mathrm{S}_{1}$ et par $\mathrm{S}_{2}$. Si de plus $\mathrm{S}_{1}$ et $S_{2}$ ont même intensité, les tensions qui apparaissent aux bornes de $\mathrm{C}_{1}$ et $\mathrm{C}_{2}$ considérées séparément (et non plus seulement leur différence comme dans le montage précédent) ne renferment pas de composante

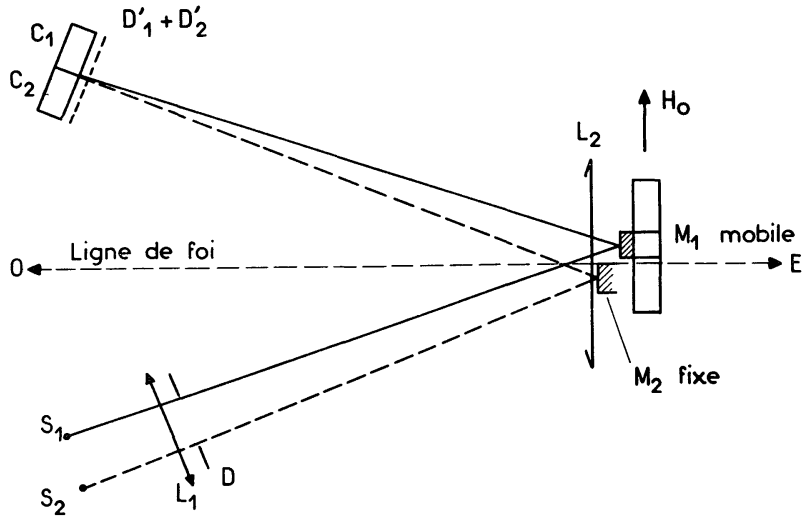

FIG. 3.

à la fréquence de modulation. Au voisinage de ce réglage, la variation de $\sigma$ avec $\theta$ n'intervient plus qu'au deuxième ordre et la principale cause de dérive se trouve ainsi éliminée. L'amélioration par rapport à un appareil normal peut atteindre un facteur 10, parfois davantage.

Les performances que nous avons pu obtenir avec ce dispositif sont les suivantes :

A court terme dans une bande passante allant de 0 à $0,2 \mathrm{c} / \mathrm{s}$, la sensibilité utilisable est environ $0,01 \gamma$. La dérive thermique sous réserve d'un échauffement uniforme du capteur est trop faible pour être mesurée avec précision. Elle est au plus de $0,003 \gamma /{ }^{\circ} \mathrm{C}$. La stabilité à long terme est de l'ordre de $0,02 \gamma$ par 24 heures et $0,1 \gamma$ sur plusieurs semaines, ceci sans aucun dispositif thermostatique. Dans des conditions climatiques contrôlées, la stabilité est certainement plus grande encore. Par contre, si le capteur est soumis à un gradient thermique, des dérives importantes peuvent se manifester par suite de la déformation du bâti métallique. Tous ces résultats ont été déterminés par comparaison entre deux appareils identiques placés côte à côte. Ils représentent la plus grande variation aléatoire d'origine non magnétique observable dans un intervalle de temps donné, l'amplitude de cette variation (exprimée en $\gamma$ ) étant arbitrairement choisie comme limite de la sensibilité du magnétomètre. De même la bande passante est définie comme étant celle où la sensibilité ainsi déterminée conserve une valeur au moins égale à $50 \%$ de sa valeur maximum.

L'appareil réel se compose d'un capteur qui peut être placé sous vide pour éviter les gradients thermiques et les mouvements de convection de l'air. Les circuits électroniques constitués en grande partie de circuits intégrés comportent, en plus de ceux déjà décrits, divers dispositifs annexes assurant la réasservissement (en cas de panne d'alimentation), le changement automatique de la gamme de mesure et la conversion du signal électrique de sortie en une variation de fréquence, ce qui permet soit l'enregistrement sur bande magnétique, soit la transmission radio de l'information. L'électronique est placée dans une enceinte étanche à l'humidité et reliée au capteur par un câble multiconducteur d'une vingtaine de mètres de long. L'alimentation s'effectue à partir d'une alimentation stabilisée $+6,0,-6 \mathrm{~V}$ ou de batteries d'accumulateurs. La dérive en fonction de la tension d'alimentation est d'environ $0,5 \gamma / \mathrm{V}$. La puissance nécessaire est de $8 \mathrm{~W}$ dont la plus grande partie est consommée dans la source. 